\title{
Composition of Metabolites in Swamp Rice Varieties to Stress Tolerance Screening
}

\author{
Sujinah*, Swisci Margaret, Indrastuti Apri Rumanti, Nurwulan Agustiani \\ Indonesian Center for Rice Research, Indonesian Agency for Agricultural Research and Development, Subang, Indonesia
}

Email address:

sujinahsulaiman26@gmail.com (Sujinah)

${ }^{*}$ Corresponding author

\section{To cite this article:}

Sujinah, Swisci Margaret, Indrastuti Apri Rumanti, Nurwulan Agustiani. Composition of Metabolites in Swamp Rice Varieties to Stress Tolerance Screening. Journal of Plant Sciences. Vol. 9, No. 3, 2021, pp. 113-118. doi: 10.11648/j.jps.20210903.16

Received: May 24, 2021; Accepted: June 9, 2021; Published: June 22, 2021

\begin{abstract}
Rice is an important food and produces metabolites which play several roles in the defense of the plant against abiotic and biotic stress. The development of stress tolerance variety is an importance for rice production. This study aims to analyze the metabolites of rice straw extract using Gas Chromatography-Mass Spectrometry (GC-MS). The analysis was carried out with 10 rice straw extracts at the flowering stage, while a total of 78 compounds were identified from the extract. The number of compounds in each variety ranged from $12-23$. Fatty acid was the most dominant compound with $37 \%$, followed by ester $26 \%$, and steroid $14 \%$. Palmitic, linoleic, and oleic acid were fatty acids found in many varieties. In addition, ethyl palmitate and 2-ethylhexyl hydrogen phthalate were also detected in all extracts. Based on the results, the highest content found in all varieties was 2-ethylhexyl hydrogen phthalate, except for Inpara 7 and 8 which predominantly contain linoleic acid. Inpara 4, 3, and 5 were included in one cluster that has a higher 2-ethylhexyl hydrogen phthalate, but a lower palmitic and linoleic acid content. The fatty acid can be used as one of the potential criteria in screening varieties for tolerance to stress, especially cold temperature, salinity, and blast disease.
\end{abstract}

Keywords: Swamp Rice, Metabolite, Tolerance, Abiotic and Biotic Stress

\section{Introduction}

Rice is an important food crop, consumed by the world's population, its production is influenced by genetic and environmental factors. Abiotic and biotic are environmental factors that affect rice production by reducing crop growth and productivity. Abiotic stress adversely affects crop growth via high and low temperature, drought, salinity, submergence, acid sulfate soils, greenhouse gases, and nutritional deficiencies [1]. Whereas, biotic include insect pest, fungi, bacteria, viruses, and herbicide toxicity. These factors have a huge impact and reduce yields by more than $50 \%$. The paddy has specific mechanisms to respond to stress conditions. However, this varies depending on the severity of the stress, age of the plant, as well as inherent stress [2]. Moreover, plants produce secondary metabolites that play a role in response to changing environment [3]. These compounds induce disease resistance, anti-insect, and allelopathic activities against biotic and abiotic stress, or as plant growth regulators [4].
Secondary metabolites in rice have been identified with over 276 compounds which includes phenolic acids, flavonoids, terpenoids, steroids, alkaloids, and other derivates [5]. Phenolic acids constitute an important class in the phenolic fraction, and some of the derivatives detected in rice include salicylic, 4-hydroxybenzoic, gallic, vanillic, p-coumaric, caffeic, and ferulic acid, etc. Primary metabolites have a strong correlation with phenolic acids, and phenylalanine was found to be most significant in making phenolics prediction model for rice grain [6]. Furthermore, the major terpenoid compounds found in rice include limonene, trans- $\beta$-ocimene, $\beta$-cymene, and linalool [7]. The constitutive emissions of $\mathrm{E} \beta \mathrm{f}$ and limonene regulated by the constitutive expression of tps 46 tends to play a crucial role in rice defense against Rhopalosiphum padi [8]. Meanwhile, accumulation of sterols, such as campesterol, $\beta$-sitosterol, and stigmasterol play a role in drought tolerance [9].

The identification of compound's content is done by 
various methods, including GC-MS (Gas Chromatography-Mass Spectrometry). GC-MS is used as a metabolomics analytical method due to its excellent chromatographic separation [10], high sensitivy and resolution [11]. This method is widely used as a separation technique for volatile organic molecules such as hydrocarbons, alcohols, steroids, fatty acids, etc. This study aims to identify secondary metabolites present in straw rice. The study outcomes provide an alternative in choosing varieties based on the content of for specific purposes, especially to abiotic and biotic stress tolerance.

\section{Materials and Methods}

\subsection{Materials}

A total of 10 swampy rice varieties were used including Inpara 1, 2, 3, 4, 5, 6, 7, Inpara 8 and 9 Agritan, as well as Inpara $10 \mathrm{BLB}$. All plants were cultivated under the same condition at Screen House, Indonesian Center for Rice Research, Indonesia. The stem and leaves were taken at the flowering stage and then oven-dried at $35^{\circ} \mathrm{C}$ and mashed while the sample was macerated using methanol. The injection of each sample was $5 \mu$ l.

\subsection{Analysis of GCMS}

Gas chromatography analysis was performed using Agilent Technologies 7890 with autosampler and 5975 mass selective detector as well as chemstation data system. The column was a $30 \mathrm{~m} \times 0.20 \mathrm{~mm}$ i.d $\times 0.11 \mu \mathrm{m}$ film thickness HP Ultra with an initial temperature of $80^{\circ} \mathrm{C}$ for 0 minutes, increased at $3^{\circ} \mathrm{C} / \mathrm{min}$ to $150^{\circ} \mathrm{C}$ for 1 minute, and then $20^{\circ} \mathrm{C} / \mathrm{min}$ to $280^{\circ} \mathrm{C}$ for 26 minutes. Furthermore, the flow rate of helium $(\mathrm{He})$ carrier gas was set at $1.2 \mathrm{ml} / \mathrm{min}$ while the temperature of the ion source was $230^{\circ} \mathrm{C}$ and the electron was set at $70 \mathrm{eV}$ using electron impact ionization method. Compound identification was carried out using mass spectra library search and the results from this study were compared with the data from the literature.

\section{Result and Discussion}

Based on the GCMS analysis, the rice straw was identified to contain 78 compounds, however, the number of compounds for each variety was different. The highest was found in Inpara 2 (23 compounds), while the lowest was in Inpara 8 (12 compounds). Furthermore, the number of compounds found in Inpara 1, 3, 4, 5, 6, 7, 9, and 10 were 17, 17, 16, 22, 19, 21, 19, and 22 respectively (Table 1 ). Chemical compounds of a plant are affected by biotic and abiotic stress, however, the biosynthetic regulation of rice compounds is highly complex [4] and strictly regulated at the transcriptional, translation, and metabolomic levels [12]. Among distinct compounds, the peaks and metabolite were identified by comparing the mass spectra and retention times with the standard. Eight rice straw extracts (Inpara 1, 3, 4, 5, 6, 7, 9, and 10) were shown to have peaks at the same compounds, 2-ethylhexyl hydrogen phthalate (Phthalic acid, mono-(2-ethylhexyl) ester). Meanwhile, two extracts (Inpara 7 and 8) have peaks in linoleic acid. Phthalic acid is an aromatic dicarboxylic acid. 2-ethylhexyl hydrogen phthalate (MEHP) is an active metabolite of bis(2-ethylhexyl) phthalate (DEHP) and its hydrolyzes via the enzyme bis(2-ethylhexyl) phthalate acylhydrolase. Linoleic acid is an essential polyunsaturated fatty acid and precursor to oxidized product by auto oxidation or enzimatically via lipoxygenase (LOX), cyclooxygenase (COX), cytochrome P450 (cyp450), and soluble epoxide hydrolase (sEH) [13].

The compounds were classified into nine classes: alcohol, aldehyde, terpenoid, ester, fatty acid, hydrocarbon, ketone, and steroid and others (Figure 1). Rice straw extract was dominated by fatty acids $37 \%$, followed by ester $26 \%$ and steroid $14 \%$. Fatty acid dominated the components in 8 rice straw extracts i.e Inpara 1, 2, 4, 6, 7, 8, 9, and 10. However, Inpara 3 and 5 were dominated by esters. Along with other derivates it plays crucial roles in plant defense responses. In addition, fatty acid is a potential molecular marker useful for basic studies on cold tolerance [14]. Salinity stress triggers the biosynthesis of fatty acid, as certain content such as palmitic, stearic, oleic, and linoleic acid were higher under salinity stress compared to control at the vegetative stage, but lower at ripening stage [15]. Suppressing the gene for stearoyl acyl carrier protein fatty acid desaturase (SACPD) resulted in increased resistance to blast and leaf blight diseases in rice [16].

Tabel 2 shows the five most abundant compounds detected by GC-MS. All rice straw extracts contained 2-ethylhexyl hydrogen phthalate, while palmitic acid was also shown to be present in all extracts except Inpara 3. The phthalic acid esters are widely used in the medical, cosmetics, paint, and pesticide industries. Meanwhile, the accumulation of (2-ethylhexyl) phthalate (DEHP) in the soil induce adverse effects on plant and pose a risk to human health [17]. DEHP had toxic effects on physiological and biochemical parameters characterized by decreased chlorophyll and increased MDA content, altered levels of antioxidant system enzymes, and decreased soluble sugar and vitamin C content in Brassica chinensis [18]. Fatty acids were also found in rice bran, with the majority being palmitic, oleic, linoleic, and linolenic acid [19]. The unsaturated fatty acids act as ingredients and modulators of cellular membranes glycerolipids, a reserve of carbon and energy in triacylglycerol, precursors of bioactive molecules, regulators of stress signaling, and inducers of oxidative stress [20]. Also, oleic and linoleic acids were reported to be involved in regulated development, seed colonization, and mycotoxin production by Aspergillus spp [21]. 
Table 1. Metabolites of swamp rice straw extracts.

\begin{tabular}{|c|c|c|c|c|c|c|c|c|c|c|c|}
\hline No & Compound & V1 & $\mathbf{V 2}$ & V3 & $\mathrm{V4}$ & V5 & V6 & V7 & V8 & V9 & V10 \\
\hline 1 & Phytone & + & + & + & + & + & + & + & + & - & + \\
\hline 2 & Ethyl palmitate & + & + & + & + & + & + & + & + & + & + \\
\hline 3 & Palmitic acid & + & + & + & + & + & + & + & + & + & + \\
\hline 4 & Cysteamine S-sulfate & + & - & - & - & - & - & - & - & - & - \\
\hline 5 & Linoleic acid & + & + & - & - & - & + & + & + & + & + \\
\hline 6 & 2-Octylcyclopropaneoctanal & + & + & - & - & - & - & - & - & - & - \\
\hline 7 & 9,17-Octadecadienal & + & + & + & - & - & - & - & - & - & - \\
\hline 8 & 2-Octadecoxyethanol & + & + & - & - & - & - & - & - & - & - \\
\hline 9 & 2-ethylhexyl hydrogen phthalate & + & + & + & + & + & + & + & + & + & + \\
\hline 10 & Heptadecyl bromide & + & - & - & - & - & - & - & - & - & - \\
\hline 11 & Lignoceric acid methyl ester & + & + & - & - & - & - & - & - & - & - \\
\hline 12 & Oleic acid & + & + & - & + & + & + & + & - & + & - \\
\hline 13 & Pyridine-3-carboxamide, oxime, N-(2-trifluoromethylphenyl)- & + & - & - & + & - & + & - & - & - & - \\
\hline 14 & Z-14-nonacosane & + & - & - & - & - & - & - & - & - & - \\
\hline 15 & 5,6-Dihydroergosterol & + & - & - & - & - & - & - & + & - & - \\
\hline 16 & Tremulone & + & + & - & - & + & + & + & + & - & + \\
\hline 17 & Sitostenone & + & & + & + & + & - & - & - & + & + \\
\hline 18 & Glycerin & - & + & + & + & + & + & - & + & - & - \\
\hline 19 & Iodooctadecane & - & + & - & - & - & - & - & - & - & - \\
\hline 20 & 9-Octadecenoic acid & - & + & - & - & - & - & - & - & - & - \\
\hline 21 & (14ß)-Pregnane & - & + & - & + & - & - & - & - & - & - \\
\hline 22 & 4,4-dimethylcholestan-3-one & - & + & - & - & - & - & - & - & - & - \\
\hline 23 & Asclepic acid & - & + & + & - & - & + & - & - & - & - \\
\hline 24 & Cholesta-6,22,24-triene,4,4-dimethyl- & - & + & - & - & - & - & - & - & - & - \\
\hline 25 & Campesterol & - & + & - & - & + & + & - & - & - & - \\
\hline 26 & Stigmasta-5,22-dien-ol & - & + & - & - & - & & - & - & - & - \\
\hline 27 & g-Sitosterol & - & + & - & - & - & + & + & - & - & + \\
\hline 28 & Cholest-4-ene-3,24-dione & - & + & - & + & - & - & - & - & - & + \\
\hline 29 & 4,22-stigmastadiene-3-one & - & + & + & - & - & - & - & - & + & + \\
\hline 30 & Neophytadiene & - & - & + & - & + & - & + & - & - & - \\
\hline 31 & 2-methyltetralin & - & - & + & - & - & - & - & - & - & - \\
\hline 32 & 2-methyl-Z-Z-,13-octadecadienol & - & - & + & - & - & - & - & - & - & - \\
\hline 33 & Oleamid & - & - & + & - & + & - & - & - & - & - \\
\hline 34 & Cholestan-3-one,4,4-dimethyl-,(5.alpha.)- & - & - & + & - & - & - & - & - & - & - \\
\hline 35 & Stigmasterol & - & - & + & + & + & + & + & + & + & + \\
\hline 36 & Ergost-4-en-3-one,(24R)- & - & - & + & - & + & - & - & - & + & - \\
\hline 37 & 13-octadecenal & - & - & + & - & - & - & - & - & - & - \\
\hline 38 & Nopinone & - & - & - & + & - & - & - & - & - & - \\
\hline 39 & (Z)-9,17-Octadecadienal & - & - & - & + & - & - & - & - & + & + \\
\hline 40 & 9-Octadecenoic acid & - & - & - & + & + & - & - & - & - & - \\
\hline 41 & Aspidospermidin-17-ol, 1-acetyl-19,21-epoxy-15,16-dimethoxy- & - & - & - & + & + & + & - & - & - & - \\
\hline 42 & $\beta$-sitosterol & - & - & - & + & + & + & - & + & + & + \\
\hline 43 & 5,5-Dimethyl-3-heptyne & - & - & - & - & + & - & - & - & - & - \\
\hline 44 & $2(5 \mathrm{H})$-furanone, 5-ethyl- & - & - & - & - & + & - & - & - & - & - \\
\hline 45 & 4,6-cholestadienol & - & - & - & - & + & - & - & - & - & - \\
\hline 46 & Propargyl alcohol, trifluoroacetate & - & - & - & - & + & - & - & - & - & - \\
\hline 47 & Ethyl vallesiachotamate & - & - & - & - & + & - & - & - & - & - \\
\hline 48 & Lauryl palmitate & - & - & - & - & + & - & - & - & - & - \\
\hline 49 & Z,Z-10,12-Hexadecadien-1-ol acetate & - & - & - & - & - & + & - & - & - & - \\
\hline 50 & Heptacosane,1-chloro & - & - & - & - & - & + & - & - & - & - \\
\hline 51 & Nonacosane & - & - & - & - & - & + & - & - & - & - \\
\hline 52 & Stigmastryl toslate & - & - & - & - & - & + & + & - & - & - \\
\hline 53 & Pentadecanoic acid & - & - & - & - & - & - & + & - & - & - \\
\hline 54 & 10-methyl-9-nonadecene & - & - & - & - & - & - & + & - & - & - \\
\hline 55 & 1,2-15,16-diepoxyhexadecane & - & - & - & - & - & - & + & - & - & - \\
\hline 56 & Ethyl oleate & - & - & - & - & - & - & + & - & - & - \\
\hline 57 & trans squalene & - & - & - & - & - & - & + & - & - & - \\
\hline 58 & Z-14-nonacosane & - & - & - & - & - & - & + & - & - & - \\
\hline 59 & 14-methylcholesta-2,8-dien-6-yl acetate & - & - & - & - & - & - & + & - & - & - \\
\hline
\end{tabular}




\begin{tabular}{|c|c|c|c|c|c|c|c|c|c|c|c|}
\hline No & Compound & V1 & V2 & V3 & V4 & V5 & V6 & V7 & V8 & V9 & V10 \\
\hline 60 & 3-Eicosene,(E)- & - & - & - & - & - & - & + & - & - & - \\
\hline 61 & 11,13-Dimethyl-12-tetradecen-1-ol acetate & - & - & - & - & - & - & + & - & - & - \\
\hline 62 & Cyclopropaneoctanal acid & - & - & - & - & - & - & + & + & - & - \\
\hline 63 & Heptadecanoic acid & - & - & - & - & - & - & - & + & + & - \\
\hline 64 & 3-ethoxy-1,2-propanediol & - & - & - & - & - & - & - & - & + & - \\
\hline 65 & (2S,5R)-2-isopropyl-5 methylhept-6-en-1-ol & - & - & - & - & - & - & - & - & + & - \\
\hline 66 & Elaidic acid ethyl ester & - & - & - & - & - & - & - & - & + & - \\
\hline 67 & Ethyl margarate & - & - & - & - & - & - & - & - & + & + \\
\hline 68 & Ethyl tetradecanoate & - & - & - & - & - & - & - & - & + & - \\
\hline 69 & 1,3-dioxane,2,2,4,5-tetramethyl-6-(1-methyloctadecyl)- & - & - & - & - & - & - & - & - & + & - \\
\hline 70 & 4-vinylcholesta-3-ol & - & - & - & - & - & - & - & - & + & - \\
\hline 71 & Erythritol & - & - & - & - & - & - & - & - & - & + \\
\hline 72 & Monoelaidin & - & - & - & - & - & - & - & - & - & + \\
\hline 73 & Eicosane & - & - & - & - & - & - & - & - & - & + \\
\hline 74 & Nonadecane & - & - & - & - & - & - & - & - & - & + \\
\hline 75 & Octacosyl acetate & - & - & - & - & - & - & - & - & - & + \\
\hline 76 & 1,3-cyclohexadecanedione & - & - & - & - & - & - & - & - & - & + \\
\hline 77 & 13-Methyl-Z-14-nonacosane & - & - & - & - & - & - & - & - & - & + \\
\hline 78 & Cyclo laudenol & - & - & - & - & - & - & - & - & - & + \\
\hline
\end{tabular}

Note: $\mathrm{V} 1=$ Inpara 1, V2=Inpara 2, V3=Inpara 3, V4= Inpara 4, V5= Inpara 5, V6= Inpara 6, V7= Inpara 7, V8= Inpara 8, V9= Inpara 9, V10= Inpara 10 $(+)$ indicated the compound was detected, (-) indicated the compound wasn't detected

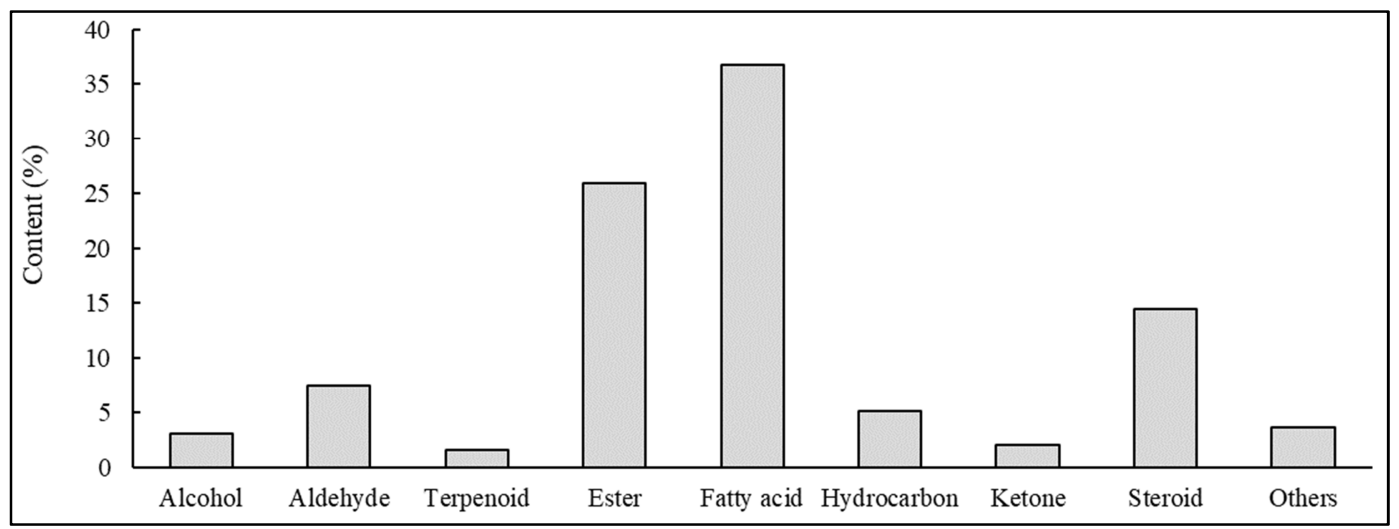

Figure 1. The compound content of swamp rice straw extract.

Table 2. Top five compounds in swamp rice straw extract.

\begin{tabular}{|c|c|c|c|c|c|}
\hline No & Inpara 1 & Inpara 2 & Inpara 3 & Inpara 4 & Inpara 5 \\
\hline 1 & $\begin{array}{l}\text { 2-ethylhexyl hydrogen } \\
\text { phthalate }\end{array}$ & $\begin{array}{l}\text { 2-ethylhexyl hydrogen } \\
\text { phthalate }\end{array}$ & $\begin{array}{l}\text { 2-ethylhexyl hydrogen } \\
\text { phthalate }\end{array}$ & $\begin{array}{l}\text { 2-ethylhexyl hydrogen } \\
\text { phthalate }\end{array}$ & $\begin{array}{l}\text { 2-ethylhexyl hydrogen } \\
\text { phthalate }\end{array}$ \\
\hline 2 & Linoleic acid & Palmitic acid & Stigmast-4-en-3-one & Oleic acid & Glycerin \\
\hline 4 & Z-14-Nonacosane & (14 $\beta$ )-Pregnane & Glycerin & 9-Octadecenoic acid & Ergost-4-en-3-one,(24R)- \\
\hline 5 & 9,17-Octadecadienal & 9,17-Octadecadienal & Ergost-4-en-3-one,(24R)- & (14 $\beta)$-Pregnane & Stigmast-4-en-3-one \\
\hline
\end{tabular}

Table 2. Continued.

\begin{tabular}{|c|c|c|c|c|c|}
\hline No & Inpara 6 & Inpara 7 & Inpara 8 & Inpara 9 & Inpara 10 \\
\hline 1 & $\begin{array}{l}\text { 2-ethylhexyl } \\
\text { hydrogen phthalate }\end{array}$ & Linoleic acid & Linoleic acid & $\begin{array}{l}\text { 2-ethylhexyl hydrogen } \\
\text { phthalate }\end{array}$ & $\begin{array}{l}\text { 2-ethylhexyl hydrogen } \\
\text { phthalate }\end{array}$ \\
\hline 2 & Palmitic acid & Palmitic acid & 2-ethylhexyl hydrogen phthalate & Linoleic acid & Linoleic acid \\
\hline 3 & Oleic acid & 2-ethylhexyl hydrogen phthalate & Palmitic acid & Palmitic acid & Palmitic acid \\
\hline 4 & Linoleic acid & Z-14-nonacosane & Cyclopropaneoctanal acid & 3-ethoxy-1,2-propanediol & Octacosyl acetate \\
\hline 5 & Nonacosane & Oleic acid & Glycerin & (Z)-9,17-Octadecadienal & $\begin{array}{l}\text { Heptadecanoic acid, ethyl } \\
\text { ester }\end{array}$ \\
\hline
\end{tabular}

Furthermore, the compound contained in each extracts variety varied. There were 10 dominant compounds in 10 rice straw extracts (Tabel 3). The compounds found in all extracts include ethyl palmitate, 2-ethylhexyl hydrogen phthalate, and palmitic acid. Meanwhile, the highest average concentration was found in 2-ethylhexyl hydrogen phthalate (20.35), followed by linoleic (15.04) and palmitic acid (12.41). Compounds with less than $5 \%$ prevalence include glycerin (3.74), ethyl palmitate (2.59), phytone (1.94), tremulone (1.42), stigmasterol (2.84), and $\beta$ sitosterol (2.60). Secondary 
metabolites are widely used as chemicals for drugs, flavors, fragrances, insecticides, dyes, and have a great economic value [22]. For example, glycerin is used in the medical, pharmaceutical, cosmetic, and food industry. In addition, rice bran glycerin extract is potentially useful as natural antidiabetic and antiglycation [23]. The appressoria of rice blast fungus (Magnaporthe grisea) reportedly used glycerin to generate pressure which ruptures plant cuticles [24] and collectively drive turgor penetration of the rice leaf [25].

Heatmap and cluster analysis was used to classify varieties and compounds according to content. Cluster analysis was carried out using average linkage with Euclidean distance. Figure 2 showed two clusters varieties, the first cluster has three varieties, i.e Inpara 4, 3, and 5 whereas the second cluster consist of seven varieties, i.e Inpara 8, 7, 6, 1, 2, 9, and 10. Based on compound content, 2-ethylhexyl hydrogen phthalate was the highest and was included in one cluster while palmitic and linoleic acid belong to the same cluster. Both compounds were included in fatty acids group with $\mathrm{C} 16$ and $\mathrm{C} 18$ respectively.

Table 3. The dominant compounds of the swamp rice straw extract.

\begin{tabular}{|c|c|c|c|c|c|c|c|c|c|c|c|}
\hline \multirow{2}{*}{ Compounds } & \multirow{2}{*}{ Formula } & \multicolumn{10}{|c|}{ Peak area $(\%)$} \\
\hline & & V1 & $\mathbf{V 2}$ & $\mathbf{V 3}$ & V4 & V5 & V6 & V7 & V8 & V9 & V10 \\
\hline Glycerin & $\mathrm{C}_{3} \mathrm{H}_{8} \mathrm{O}_{3}$ & - & 2.09 & 6.56 & 1.04 & 8.38 & 1.72 & - & 2.64 & - & - \\
\hline Ethyl palmitate & $\mathrm{C}_{18} \mathrm{H}_{36} \mathrm{O}_{2}$ & 3.42 & 3.12 & 1.49 & 3.73 & 1.63 & 3.32 & 2.30 & 2.39 & 2.37 & 2.14 \\
\hline 2-ethylhexyl hydrogen phthalate & $\mathrm{C}_{16} \mathrm{H}_{22} \mathrm{O}_{4}$ & 20.19 & 16.03 & 34.76 & 17.74 & 31.36 & 15.40 & 12.23 & 22.67 & 18.55 & 14.61 \\
\hline Palmitic acid & $\mathrm{C}_{16} \mathrm{H}_{32} \mathrm{O}_{2}$ & 16.12 & 14.67 & 3.60 & 14.18 & 5.42 & 14.77 & 15.14 & 20.57 & 7.97 & 11.67 \\
\hline Linoleic acid & $\mathrm{C}_{18} \mathrm{H}_{32} \mathrm{O}_{2}$ & 17.52 & 8.41 & - & - & - & 10.34 & 17.23 & 26.43 & 11.83 & 13.52 \\
\hline Phytone & $\mathrm{C}_{18} \mathrm{H}_{36} \mathrm{O}$ & 2.18 & 1.68 & 1.43 & 2.02 & 1.54 & 1.91 & 2.19 & 2.63 & - & 1.88 \\
\hline Tremulone & $\mathrm{C}_{29} \mathrm{H}_{46} \mathrm{O}$ & 1.55 & 1.15 & - & - & 1.95 & 1.16 & 1.25 & 1.06 & - & 1.84 \\
\hline Stigmasterol & $\mathrm{C}_{29} \mathrm{H}_{48} \mathrm{O}$ & - & - & 2.69 & 1.19 & 3.05 & 3.05 & 4.62 & 2.03 & 2.33 & 3.75 \\
\hline$\beta$ sitosterol & $\mathrm{C}_{16} \mathrm{H}_{22} \mathrm{O}_{4}$ & - & - & - & 1.48 & 3.31 & 2.50 & - & 2.40 & 2.35 & 3.56 \\
\hline
\end{tabular}

Note: V1= Inpara 1, V2=Inpara 2, V3=Inpara 3, V4= Inpara 4, V5= Inpara 5, V6= Inpara 6, V7= Inpara 7, V8= Inpara 8, V9= Inpara 9, V10= Inpara 10

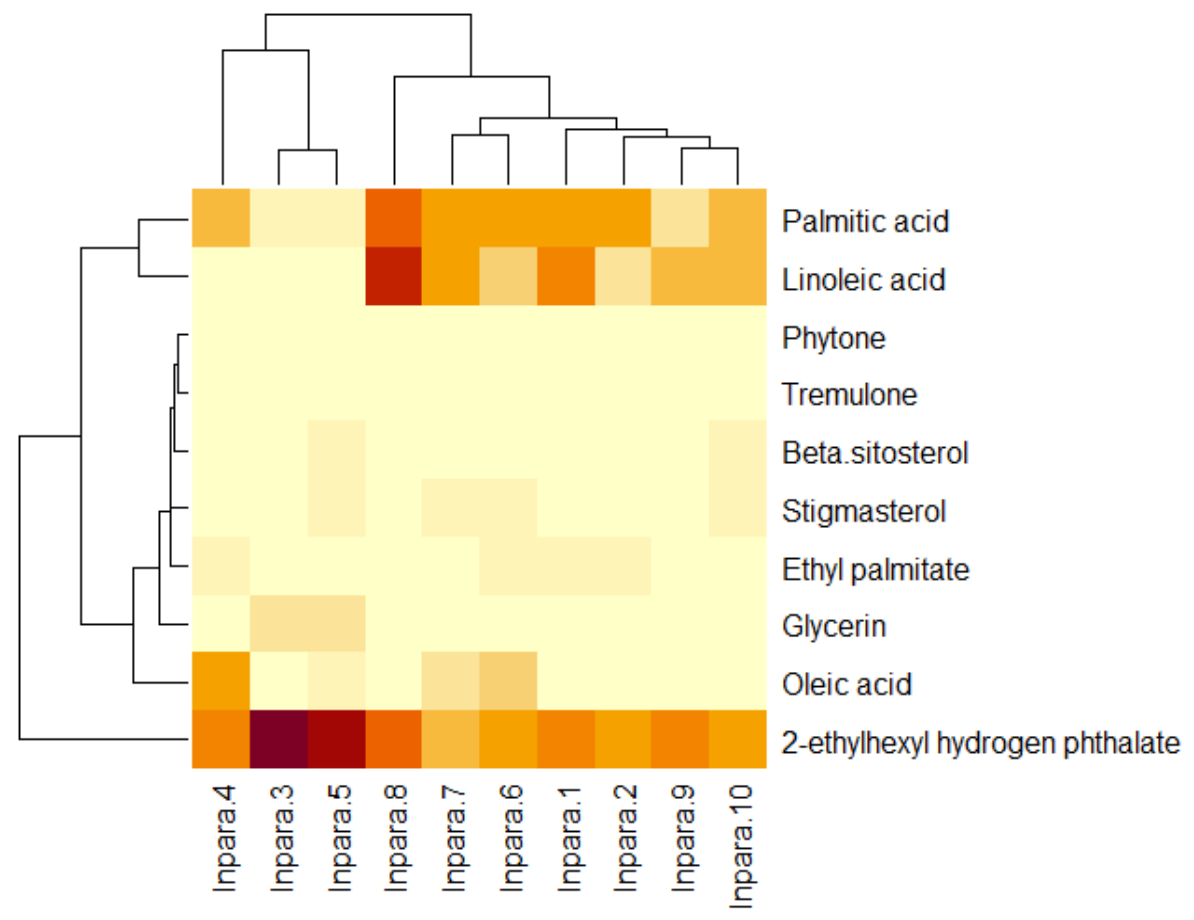

Figure 2. Heatmap and cluster analysis of the compound in swamp rice straw extract.

\section{Conclusion}

Based on the result, rice straw extract contains different amounts of metabolites, ranging between 12 to 23 compounds. The identified compounds were categorized into several groups which include alcohol, aldehyde, terpenoid, ester, fatty acid, hydrocarbon, ketone, steroid and others. 2-ethylhexyl hydrogen phthalate was the most dominant compound in all varieties, except Inpara 7 and 8 which predominantly contain linoleic acid. The fatty acids are potential metabolites useful for screening of rice tolerance to cold temperature, salinity, and blast disease. 


\section{Acknowledgements}

The authors are grateful to all those who helped in course of the research, especially Endin.

\section{References}

[1] Singh T, Pun K B, Saikia K, Satapathy BS, Bhagat K, Das A, Lal B. (2015). Abiotic stress management in rice, In: BJ Rajkhowa, A Das, SV Ngachan, AK Sikka, and M Lyngdoh (Eds.), Integrated Soil and Water Resource Management for Livelihood and Environmental Security. ICAR Research Complex for NEH Region Meghalaya: 219-258.

[2] Anami B S, Malvade N N and Palaiah S. (2020). Classification of yield affecting biotic and abiotic paddy crop stresses using field images. Information Processing in Agriculture 7: 272-285. DOI: 10.1016/j.inpa.2019.08.005.

[3] Berini J L, Brockman S A, Hegeman A D, Reich P B, Muthukrishnan R, Montgomery R A and Forester J D. (2018). Combinations of abiotic factors differentially alter production of plant secondary metabolites in five woddy plant species in the boreal-temperate transition zone. Frontiers in Plant Science 9 (1257): 1-17. DOI: 10.3389/fpls.2018.01257.

[4] Wang W, Li Y, Dang P, Lai D, Zhao S and Zhou L. (2018). Rice secondary metabolites: structures, roles, biosynthesis, and metabolic regulation. Molecules 23 (3098): 1-50. DOI: 10.3390/molecules23123098.

[5] Ciulu M, de la Luz Cadiz-Gurrea M and Segura-Carretero A. (2018). Extraction and analysis of phenolic compounds in rice: a review. Molecules 23 (2890): 1-20. DOI: 10.3390/molecules23112.

[6] Kim J K, Park S Y, Lim S H, Yeo Y, Choand H S and Ha SH. (2013). Comparative metabolic profiling of pigmented rice (Oryza sativa L.) cultivars revelas primary metabolites are correlated with secondary metabolites. Journal of Cereal Science 57: 14-20. DOI: 10.1016/j.jcs.2012.09.012.

[7] Chumpolsri W, Wijit N, Boontakham P, Nimmanpipug P, Sookwong P, Luangkamin S and Wongpornchai S. (2015). Variation of terpenoid flavor odorants in brand of some black and white varieties analyzed by GCxGC-MS. Journal of Food and Nutrition Research 3 (2): 114-120. DOI: 10.12691/jfnr-3-2-7.

[8] Sun Y, Huang X, Ning Y, Jing W, Bruce T J A, Qi F, Xu Q, Wu K, Zhang Y and Guo Y. (2017). TPS46, a rice terpenne synthase conferring natural resistance to birdcherry-oat aphid, Rhopalosiphum padi (Linnaeus). Frontiers in Plant Science 8 (110): 1-11. DOI: 10.3389/fpls.2017.00110.

[9] Kumar M S S, Ali K, Dahuja A and Tyagi A. (2015). Role of phytosterols in drought stress tolerance in rice. Plant Physiology and Biochemistry: 1-22. DOI: 10.1016/j.plaphy.2015.07.014.

[10] Krone N, Hughes B A, Lavery G G, Stewart P M, Arlt W and Shackleton C H L. (2010). Gas chromatography/mass spectrometry (GC/MS) remains pre-eminent discovery tool in clinical steroid investigations even in the era of fast liquid chromatography tandem mass spectrometry (LC/MS/MS). Journal of Steroid Biochemistry and Molecular Biology 121: 496-504. DOI: 10.1016/j.jsbmb.2010.04.010.

[11] Zhang L, Yu Y and Yu R. (2020). Analysis of metabolites and metabolic pathways in three maize (Zea mays L.) varieties from the same origin using GC-MS. Nature Research 10 (17990): 1-10. DOI: 10.1038/s41598-020-73041-z.

[12] Kusano M, Yang Z, Okozaki Y, Nakabayashi R, Fukushima A and Saito K. (2015). Using metabolomic approaches to explore chemical diversity in rice. Molecular Plant 8: 58-67. DOI: 10.1016/j.molp.201411.010.

[13] Taha A Y. (2020). Linoleic acid-good or bad for the brain? NJP Science of Food 4 (1): 1-6. DOI: 10.1038/s41538-019-0061-9.

[14] da Cruz R P, Golombieski J I, Bazana M T, Cabreira C, Silveira T F and da Silva L P. Alterations in fatty acid composition due to cold exposure at the vegetative stage in rice. Braz. J. Plant Physiol. 22 (3): 199-207. DOI: 10.1590/S1677-04202010000300007.

[15] Aziz A, Fairuz M S, Abdullah M Z, Ma N L and Marziah M. (2015). Fatty acid profile of salinity tolerant rice genotypes grown on saline soil. Malays. Appl. Biol. 44 (1): 119-124.

[16] Jiang C, Shimono M, Maeda S, Inoue H, Mori M, Hasegawa M, Sugano S and Takatsuji H. (2009). Suppression of the rice fatty-acid desaturase gene OsSIS2 enhances resistance to blast and leaf blight diseases in rice. Molecular Plant Microbe Interactions 22 (7): 820-829. DOI: 10.1094/ MPMI $-22-7-0820$

[17] Chen N, Shuai W, Hao X, Zhang H, Zhou D, Gao J. (2017). Contamination of phthalate esters in vegetable agriculture and human cumulative risk assessment. Pedosphere 27 (3): 439-451. DOI: 10.1016/S1002-0160(17)60340-0.

[18] Yuan L, Cheng J, Wang Y, Liu Y, Wang W, Gao R and Yu X. (2020). Uptake and toxicity of di-(2-ethylhexyl) phthalate in Brassica chinensis L. Chemosphere. 252 (126640): 1-8. DOI: 10.1016/j.chemosphere.2020.126640.

[19] Goffman F D, Pinson S and Bergman C. (2003). Genetic diversity for lipid content and fatty acid profile in rice bran. Journal of the American Oil Chemists Society 80 (5): 485-490. DOI: $10.1007 / \mathrm{s} 11746-003-0725-\mathrm{x}$.

[20] He M and Ding N Z. (2020). Plant unsaturated fatty acids: multiple roles in stress response. Frontiers in Plant Science 11 (562785): 1-15. DOI: 10.3389/fpls.2020.562785.

[21] Upchurch R G. (2008). Fatty acid unsaturation, mobilization, and regulation in the response of plants to stress. Biotechnol Lett. 30: 967-977. DOI: 10.1007/s10529-008-9639-z.

[22] Pagare R, Bhatia M, Tripathi N, Pagare S and Bansal Y K. (2015). Secondary metabolites of plants and their role: overview. Current Trends in Biotechnology and Pharmacy 9 (3): 294-305.

[23] Aalim H, Belwal T, Jiang L, Huang H, Meng X and Luo Z. (2019). Extraction optimization, antidiabetic and antiglycation potential of aquoeus glycerol extract from rice (Oryza sativa L.) bran. LWT-Food Science and Technology 103: 147-154. DOI: 10.1016/j.lwt.2019.01.006.

[24] de Jong J C, McCormack B C, Smirnoff N and Talbot N C. (1997). Glycerol generates turgor in rice blast. Nature 389: 244-245.

[25] Foster A J, Ryder L S, Kershaw M J and Talbot N J. (2017). The role of glycerol in the pathogenic lifestyle of the rice blast fungus Magnaporthe oryzae. Environmental Microbiology 19 (3): 1008-1016. DOI: 10.1111/1462-2920.13688. 